\title{
Local Crystallography for Quantitative Analysis of Atomically Resolved Images
}

Alex Belianinov, ${ }^{1,2,}$ Qian He, ${ }^{3}$ Mikhail Kravchenko, ${ }^{1,2}$ Stephen Jesse, ${ }^{1,2}$ Albina

Borisevich, ${ }^{1,3}$ Sergei V. Kalinin ${ }^{1,2}$

${ }^{1}$ Institute for Functional Imaging of Materials, Oak Ridge National Laboratory, Oak Ridge, TN 37831 .

2 The Center for Nanophase Materials Sciences, Oak Ridge National Laboratory, Oak Ridge, TN 37831 .

${ }^{3}$ Materials Sciences and Technology Division, Oak Ridge National Laboratory, Oak Ridge, TN 37831 .

High-resolution imaging in scanning transmission electron microscopies and its probe-based brethren, allows $10 \mathrm{pm}$ or better precision measurements of atomic positions. This level of fidelity is sufficient to correlate the length and energy of a chemical bond to a functional material property. By finding all the atoms in an image and analyzing their local atomic neighborhoods we can quantify and identify phases, structural defects and other properties of multiphase samples.[1] This automatic analysis lays the groundwork for processing dynamic data and amassing structure-property libraries based on the analysis of local atomic behavior.

We have utilized multivariate and clustering algorithms on multi-phase regions of various materials to identify small structural distortions, and link them to physical functionality. As a model system, we have chosen mixed oxide Mo-V-M-O $(\mathrm{M}=\mathrm{Nb}$, Ta, Te and/or Sb), which is currently the most promising catalyst for many industrially important reactions, such as propane (amm)oxidation.[2] We have also processed atomically resolved data on steel alloys and a broad range of 2D materials. Specifically, our approach returns positions of each atom in an image, in addition to local descriptors that describe the image quality in a given region. A multipronged approach to atom finding, based on a target search, sparse representation of image data, and various image transforms; is a serious improvement over the traditional peak fitting methods. [3, 4] We also explore atom tilts and image correction based on the extracted image data and metadata. [5]

Our analysis effortlessly distinguishes different areas of the image based on the similarity of chemical neighborhoods and is capable of executing this function in parallel for imaging a dynamic process. Figure 1(a) is a single $\mathrm{M} 2$ phase $\mathrm{Mo}-\mathrm{V}-\mathrm{M}-\mathrm{O}(\mathrm{M}=\mathrm{Nb}, \mathrm{Ta}, \mathrm{Te}$ and/or $\mathrm{Sb})$ STEM image that looks pristine. Figure $1(b, c)$ is the image in (a) after k-means clustering analysis for 6 neighbors, sorted by distance, and angle metrics respectively. Data from this and other material systems have been investigated, and will be presented. We believe this approach paves the way for full information recovery in high resolution imaging as well as allows classification for automatic identification of materials.[6] 

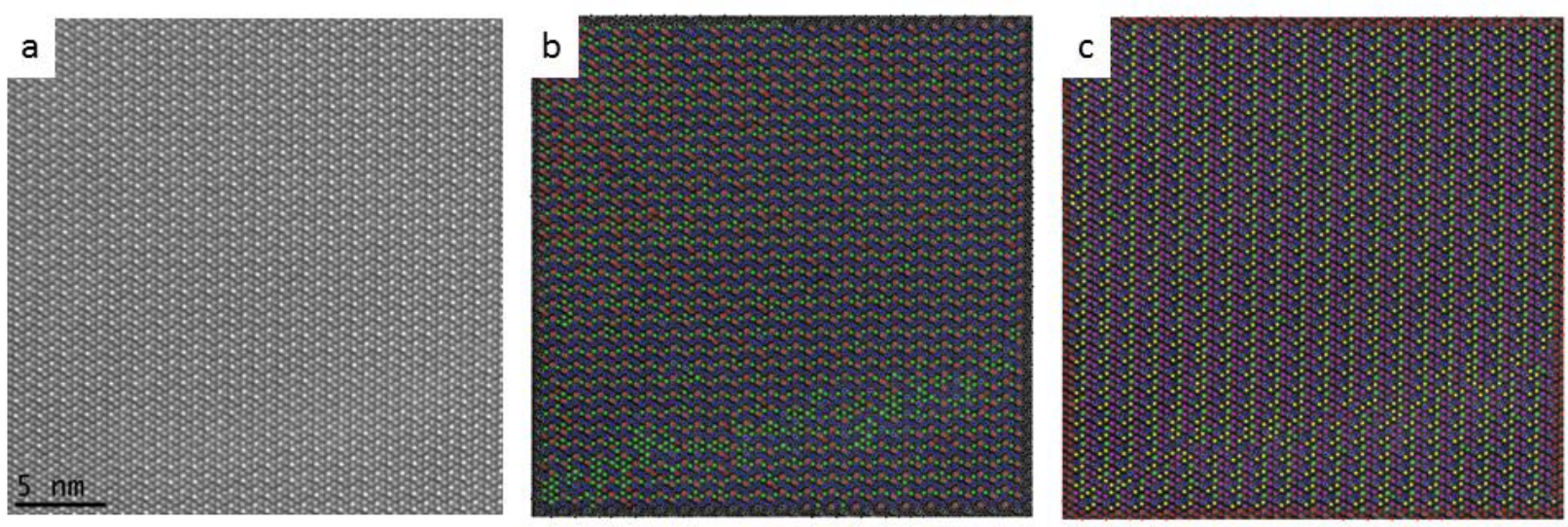

Figure 1. Single M2 phase Mo-V-M-O (M = Nb, Ta, Te and/or Sb). (a) M2 phase STEM image. (b) k-means clustering results for 6 neighbors, sorted by distance metric. (c) k-means clustering results for 6 neighbors, sorted by angle metric.

\section{References}

[1] A Belianinov, Q He, M Kravchenko, S Jesse, A Borisevich, S V. Kalinin, Identification of phases, symmetries and defects through local crystallography, Nature Communications, 6, 7801 [2] Shiju, N. R. \& Guliants, V. V. Recent developments in catalysis using nanostructured materials. Applied Catalysis A: General 356, 1-17, doi:10.1016/j.apcata.2008.11.034 (2009). [3] Xiahan Sang, Adedapo A. Oni, and James M. LeBeau, Atom Column Indexing: Atomic Resolution Image Analysis Through a Matrix Representation, Microsc. Microanal., doi:10.1017/S1431927614013506

[4] Sarahan M.C., Chi M., Masiel D.J. and Browning N.D; Point defect characterization in HAADF-STEM images using multivariate statistical analysis, Ultramicroscopy, 111 (3), pp. 251-257; 2011

[5] Jones L., Nellist P.D., Identifying and correcting scan noise and drift in the scanning transmission electron microscope (2013) Microscopy and Microanalysis, 19 (4), pp. 1050-1060. [6] Research for all authors was supported by the US Department of Energy, Basic Energy Sciences, Materials Sciences and Engineering Division. This research was conducted at the Center for Nanophase Materials Sciences, which is sponsored at Oak Ridge National Laboratory by the Scientific User Facilities Division, Office of Basic Energy Sciences, U. S. Department of Energy. 